\title{
GEOMETRY OF HYPERBOLIC 3-MANIFOLDS WITH BOUNDARY
}

\author{
By SADayoshi KoJima
}

\section{§1. Hyperbolic Manifolds with Boundary}

A hyperbolic manifold will be a complete Riemannian manifold of constant sectional curvature -1 . We call a simply connected hyperbolic manifold the hyperbolic space in particular. One standard model of the hyperbolic space is a ball $\mathbf{P}=\left\{\left(x_{1}, \cdots, x_{n}\right) \in\right.$ $\left.\mathbf{R}^{n} \mid x_{1}^{2}+\cdots+x_{n}^{2}<1\right\}$ equipped with the Poincaré metric

$$
\frac{4\left(d x_{1}^{2}+\cdots+d x_{n}^{2}\right)}{\left(1-\left(x_{1}^{2}+\cdots+x_{n}^{2}\right)\right)^{2}} .
$$

A hyperbolic manifold is, in other words, a complete Riemannian manifold locally modelled on the Poincaré ball.

Particular interests have been paid to the case in dimension 3 by many mathematicians of various fields since late 70's, probably because really significant contributions by Thurston, Gromov, Sullivan and many others justify that their mathematical structures behind appearance is undoubtedly rich. Thurston's lecture note [21] has been serving as a pioneering bible, and many other related articles such as [3,5] have appeared in these days.

In this note, we focus on more restrictive class, a hyperbolic 3-manifold of finite volume with non-empty totally geodesic boundary, and would like to report our naive studies on their geometry. Throughout the sequel, we adopt a notation $N$ to indicate such a manifold without referring conditions. $N$ will hence always mean a hyperbolic 3 -manifold of finite volume with totally geodesic boundary.

Even with these additional requirements, $N$ is still very attractive and enjoys many nice properties. At first, they are almost relatives to complete hyperbolic 3-manifolds and have many properties in common with them. They have also their own features. For example, they can be rather easily constructed, they have visible sides interplaying in geometry and topology, they are related to well established Teichmüller theory, and so on.

To advertise a flavor of naive and pleasant study of $N$, let us look at the universal covers. The universal cover $\widetilde{N}$ of $N$ is developed in $\mathbf{P}$ as a convex subset with geodesic boundary. If we see $\widetilde{N}$ from outside the Poincare ball, each component of the boundary appears much like a round hole on a golf ball surface. Its edge defines a circle on the sphere at infinity $\mathbf{P}_{\infty}=\left\{x_{1}^{2}+x_{2}^{2}+x_{3}^{2}=1\right\}$ by taking closure in the euclidean topology. $\partial \widetilde{N}$ has infinitely many components and the set of all circles appeared by closure forms a circle packing $\mathcal{C}_{N}$ on $\mathbf{P}_{\infty} \cdot \mathcal{C}_{N}$ has a completeness property due to finiteness of vol $N$,

Received June 21, 1993 
that every open component in the complement $\mathbf{P}_{\infty}-\mathcal{C}_{N}$ is a disk bounded by a circle in $\mathcal{C}_{N}$ (see [12]).

There is another way to see $\mathcal{C}_{N}$. The developed image of $\tilde{N}$ over $\mathbf{P}$ defines a faithful holonomy representation $\rho: \pi_{1}(N) \rightarrow$ Isom $\mathbf{P}$ of $\pi_{1}(N)$ describing a hyperbolic structure up to conjugacy. The image $\rho\left(\pi_{1}(N)\right)$ is a discrete group. Then the limit set of $\rho\left(\pi_{1}(N)\right)$ on $\mathbf{P}_{\infty}$, the set of accumulation points of the orbit of some point in $\mathbf{P}$ by $\rho\left(\pi_{1}(N)\right)$, is the closure of $\mathcal{C}_{N}$. Taking closure does not affect the view of packings at all.

The contact property of the packing $\mathcal{C}_{N}$ is related to the location of cusps in $N$. There are two kinds of cusps in $N$. One lies away from the boundary, which we call an int-cusp. An int-cusp is a usual toral cusp. The other is a half of a toral cusp cut by $\partial N$, which appears as a cusp on the boundary. We call such a cusp a $\partial$-cusp. The fixed point of a parabolic element generating a $\partial$-cusp must be a contact point of two circles in $\mathcal{C}_{N}$. Conversely, a contact point in $\mathcal{C}_{N}$ is a fixed point of some parabolic element generating a $\partial$-cusp. In particular, some circles in $\mathcal{C}_{N}$ meet each other iff $N$ has a $\partial$-cusp.

Consider a regular ideal octahedron, past two pairs of non-adjacent triangular faces appropriately and we obtain a $N$. The packing $\mathcal{C}_{N}$ in this case is the classical apollonian packing generated by successive inclusion of inscribed circles on the mutually tangent four circles on the plane. Consider then two regular truncated tetrahedra of dihedral angle $=\pi / 6$, which we will describe later more precisely, past their hexagonal faces appropriately and we obtain a compact $N . \mathcal{C}_{N}$ in this case is still a complete packing but no two circles are tangent any more.

The packing with non-contact property has been studied recently by a group in Mathematical Physics [4, 2] from a Möbius geometric viewpoint. However Möbius geometry is the geometry in dimension 2 and would have a little disadvantage when dealing with some global properties. Our 3-dimensional hyperbolic view shifts the problem of packings into the polyhedral geometry. In [10], we showed how our viewpoint is practically powerful to produce non-contact packings on the plane, and also theoretically manageable to study those by solving a few questions in [4].

\section{§2. Polyhedral Decomposition}

The target $N$ is a 3 -dimensional manifold at first. The dimension 3 is already too global to visualize. Hence $N$ would be expected to have a more accessible presentation, which enables to split the study into local geometry and combinations. The building block which we believe to be the best is a truncated polyhedron.

To describe truncation device more precise, we consider the Klein ball model $\mathbf{K}$ of the hyperbolic 3-space rather than the Poincaré ball. The Klein ball is the restriction of the 3-dimensional projective geometry to the unit ball. The underlying space is again the unit ball. The metric is worse described but the transformation group, the isometry group of $\mathbf{K}$, is simply the restriction of projective transformations to those who leave the unit ball invariant. One nice relation is that the sphere at infinity $\mathbf{K}_{\infty}$ of $\mathbf{K}$ is the same as $\mathbf{P}_{\infty}$.

A polyhedron of the hyperbolic 3-space in the Klein ball is the restriction of an euclidean polyhedron to $\mathbf{K}$. When an euclidean polyhedron is contained entirely in $\mathbf{K}$, it defines a compact polyhedron. If some of the vertices lie on $\mathbf{K}_{\infty}$, then the polyhedron in fact becomes non-compact, but still has finite volume. We call vertices lying on $\mathbf{K}_{\infty}$ 
ideal since they are not actual vertices.

In the ultra ideal case, some vertices of an euclidean polyhedron lie outside $\mathbf{K}_{\infty}$. Then the polyhedron automatically has infinite volume. To each outside vertex, there is a unique cone tangent to $\mathbf{K}_{\infty}$. The intersection with $\mathbf{K}_{\infty}$ is a circle and bounds a geodesic plane in $\mathbf{K}$. This plane intersects each face of the polyhedron meeting the vertex in question perpendicularly. Truncating a neighborhood of each vertices outside $\mathbf{K}_{\infty}$ by the plane appeared in this manner, we obtain a truncated polyhedron of finite volume. If the original euclidean polyhedron has no ideal vertices, then the truncated polyhedron is compact. We call by a ridge an edge joining either the faces of truncation, ideal vertices, or one and the other.

For some truncated polyhedra, we may consider a combinatorial rule for glueing them along the faces which are on the original surface and not appeared by truncation, and ask delicate geometric consistencies that come from the isometricity for each pair of identified faces, the angle condition about each ridge and the completeness for each ends. If all the requirements are satisfied, then the result of identification is now $N$ where the boundary consists of the faces of truncation. Various concrete examples are discussed in [21], and one simple class is classified in [7].

The requirement seems to be severe, but the variety of truncated polyhedra is contrary flexible. In fact, this construction will turn out to be always the case. In [6], Epstein and Penner proved that every cusped hyperbolic manifold admits a canonical decomposition by ideal polyhedra, which is dual to the Ford domain. A variant of their argument leaded us to prove

TheOREM ([12]). Every $N$ has a canonical decomposition by truncated polyhedra. If $N$ has no cusps, then it is dual to the cut locus of the boundary.

Likewise the theorem by Epstein and Penner, $N$ is not necessarily of dimension 3 . The assertion is true when the dimension is at least 2 . We gave a rather visible proof to this fact in [13] which works only in dimension 2 and 3.

\section{§3. Dehn Filling}

The non-compact complete hyperbolic manifolds of finite volume of dimension $\geqq 3$ are still dominated by Mostow rigidity $[18,20]$, that is, homotopy equivalent manifolds are isometric. This can be rephrased in terms of the representation of a fundamental group, that a discrete faithful representation to the isometry group of the hyperbolic space is unique up to conjugacy. However, the representation itself has more freedom to vary if we drop the faithfulness and discreteness conditions. In dimension 3 , this freedom was geometrically interpreted by filling an end with a slightly singular hyperbolic solid in [21, Lemma 5.8.1], (cf. [3]). These deformations are locally parametrized by a generalization of Dehn surgery coefficients in the classical knot theory. For each cusp, it is a pair of coprime integers up to sign in the classical case, and then a pair of real numbers up to sign in the new theory. We call this geometric surgery a Dehn filling

If $N$ has int-cusps, we can perform a small Dehn filling so that the boundary is being totally geodesic. To see this, take a double $D N$ and do filling with the same coefficient on each pair of cusps in $D N$ so that there is an obvious orientation reversing involution $\tau$ 
switching the sides. Let $\rho$ be the corresponding representation of $\pi_{1}(N)$ to the isometry group. If $\rho\left(\pi_{1}(\partial N)\right)$ were not conjugate to a discrete group contained in the 2-dimensional isometry group, then $\tau$ would induce a different singular hyperbolic structure from one by the Dehn filling. This contradicts the uniqueness of a small Dehn filling since both structures are geometrically near $D N$ and have the same filling coefficients. Thus the surface homotopically fixed by $\tau$ is realized as a totally geodesic surface in the filled double. Then cut it open along that surface, we obtain a Dehn filled manifold with boundary.

Let us consider only this type of small Dehn fillings on $N$, in particular, we always require the boundary to be totally geodesic. Such deformations are parametrized by the Dehn filling coefficients $\mathcal{D S}(N)$ near $N$ up to sign. $\mathcal{D S}(N)$ has complex dimension $=\#\{$ int-cusp $\}$, and can be identified locally with the subvariety of the character variety of representations of $\pi_{1}(N)$ to the isometry group of the hyperbolic 3-space.

Do a small Dehn filling on $N$. Then, assigning a new hyperbolic structure on the boundary, we get a map

$$
\partial: \mathcal{D S}(N) \rightarrow \mathcal{T}(\partial N)
$$

near $N$, where $\mathcal{T}(\partial N)$ is a Teichmüller space of $\partial N$. The Teichmüller space is a real semialgebraic variety and $\partial$ is analytic. It would be interesting to study several properties of the map $\partial$ in connection with the geometry of $N$.

There are two extremal examples. As probably the worst degenerate case, Neumann and Reid found a $N$ such that $\partial$ is a constant map in [19]. This $N$ has a special symmetry which induces a rigid property on the deformation of the boundary. As the smoothest case, Fujii worked out a calculation in [8] for a particular $N$ so that $\partial$ is an embedding near $N$. In [19], Neumann and Reid also found a computer-aided example of a 2-cusped orbifold, where small Dehn fillings on one cusp do affect the other but not in the first order. This suggests nondegenerate but possibly singular behavior of $\partial$. The situation seems to be complicated and more study is needed.

In the pioneering stage of Dehn filling theory, one concrete example is fully discussed in terms of the deformation of its tetrahedral blocks [21, §4]. There, the set of dihedral angles of tetrahedra under gluing consistency other than complete conditions parametrized Dehn fillings near the original structure. It is hence natural to carry out the same business for some $N$ based on a polyhedral decomposition.

Hence suppose that $N$ is decomposed by truncated tetrahedra. The shape of a truncated tetrahedron is determined by 6 dihedral angles at ridges, where each ideal vertex requires a 1-dimensional constrain. The gluing consistencies for $N$ in this case come from not only angle conditions around ridges but also isometricity for the glued faces. It can be shown (cf. [9]) that any small Dehn filling is performed preserving this polygonal structure effectively, so that the metric completion gives the resulting singular space. In particular, the set of dihedral angles at ridges up to gluing consistency other than completeness conditions parametrizes the space $\mathcal{D S}(N)$ near $N$.

Each image of $\partial$ is then described by a geodesic triangulation of the boundary. A geodesic triangulation contains something more than a hyperbolic structure, a point in the Teichmüller space. The ambiguity at $N$ is the freedom to vary the geodesic triangulation on $\partial N$. When we discuss the derivative of $\partial$, the local description of the freedom is sufficient. Then it is not hard to show that they are generated by perturbations of 
each vertex. These observations give us a computational frame for concrete examples at least, though no real executions have come up yet.

\section{§4. Extremal Volumes}

The volume is an obvious quantitative invariant of Riemannian manifolds. Since $N$ admits Mostow rigidity, vol $N$ becomes a homotopy invariant and represents a homotopical and in particular topological complexity numerically.

The volumes of complete hyperbolic 3-manifolds form a well ordered set of type $\omega^{\omega}$ by the theory of Jørgensen and Thurston [21]. Each limit cardinal is represented by a cusped manifold. The volumes of Dehn filled manifolds accumulate the original cusped volume from below. $\{\operatorname{vol} N\}$ has the same property. Because, since the double of $N$ is a complete manifold, the set of the volumes of $N$ 's is a subset of the half volumes of complete manifolds, and is well-ordered. Moreover, if $N$ has a cusp, then it admits Dehn fillings and the volumes accumulate the cusped one from below.

Various interesting questions can be asked, and several studies have been carried out. Among them, we would like to report our progress on finding extremal values based on Miyamoto's formulation [17], which seems to be the best approach at the moment. The idea is to find a sharp linear estimate of the vol $N$ by area $\partial N$. One negative remark is that, as was discussed in [16], the area of the face of truncation in a truncated tetrahedron can stay away from zero while its volume degenerates. Hence the existence of polyhedral decomposition does not help very much this approach.

Denote by $\Delta_{\ell}$ a regular truncated tetrahedron of all ridge lengths $=\ell . \Delta_{0}$ is a regular truncated tetrahedron with vanishing ridge length, and simultaneously a regular ideal octahedron. It can be checked by an elementary hyperbolic trigonometry that the dihedral angle $\theta$ at each ridge is determined by the equation,

$$
\cos \theta=\frac{\cosh \ell}{2 \cosh \ell-1} \text {. }
$$

$\theta$ is monotone increasing in term of $\ell$. The face of truncation is then an equilateral hyperbolic triangle of angle $=\theta$. Conversely, given an angle $0 \leqq \theta<\pi / 3$, there is a unique regular truncated tetrahedra of dihedral angle $=\theta$ at each ridge, which is isometric to $\Delta_{\ell}$. Hence we may denote $\Delta_{\ell}$ by $\Delta_{\theta}$ sometimes.

The area of $\partial N$ is related to the area of the faces of truncation on polyhedra. We use the notation "area" for a truncated polyhedron to indicate the total area of its faces of truncation rather than the total surface area. For instance, area $\Delta_{\ell}$ is the sum of the area of 4 triangular faces of truncation.

area $\Delta_{\ell}$ is obviously a function in terms of $\ell$ and hence $\theta$. On the other hand, vol $\Delta_{\ell}$ was well described by the Lobachevski function in [11]. The important ratio will be

$$
C_{\ell}=\frac{\operatorname{vol} \Delta_{\ell}}{\operatorname{area} \Delta_{\ell}}
$$

$C_{\ell}$ is monotone increasing in terms of $\ell$.

The constant $C_{\ell}$ is related to the geometry of $N$. To see this, let us introduce a return path of $N$, a geodesic segment whose end points lie on $\partial N$ such that the segment is perpendicular to the boundary at both end points. The double of a return path becomes a closed geodesic in $D N$ which perpendicularly intersects $\partial N$ at two points. It is not 
hard to show that a return path is homotopically non-trivial relative boundary, and that there are only finitely many return paths with bounded length.

If $N$ has no $\partial$-cusp, every homotopically nontrivial path relative boundary is homotopic to a unique return path. Then we let $\ell_{N}$ be the shortest length of return paths in $N$. If $N$ has no $\partial$-cusps, then there are homotopically non-trivial arbitrary short paths relative boundary tending to $\partial$-cusp. Hence let's let $\ell_{N}$ be zero in this case.

$C_{\ell_{N}}$ could be the ratio of vol $N$ and area $\partial N$ if $N$ were simply decomposed by $\Delta_{\ell_{N}}$, though such a decomposition is possible only for a very limited special class of $N$ 's. On the other hand, by studying packing in the hyperbolic space by equidistant surfaces of components of $\partial \widetilde{N}$, Miyamoto realized that this ratio is extremal, and attained only by such very special $N$ 's.

THEOREM ([17]). For every $N$,

$$
\operatorname{vol} N \geqq C_{\ell_{N}} \text { area } \partial N \text {, }
$$

holds, where the equality holds iff $N$ has a polyhedral decomposition by $\Delta_{\ell_{N}}$.

This theorem holds for dimensions $\geqq 3$ in general if we replace $\Delta_{\ell_{N}}$ by a higher dimensional truncated simplex with ridge length $=\ell_{N}$. In dimension 3 and 4 , the estimate is sharp, established by concrete examples, but no longer best possible in dimension $\geqq 5$.

In dimension 3 , the area of $\partial N$ is well known by Gauss-Bonnet formula and various sharper results can be obtained. For example, the characterization of the smallest $N^{\prime} s$, which was previously proved by an ad hoc method, is now a direct corollary since area $\partial N \geqq 4 \pi$.

COROLlaRY ([14]). Every $N$ admits the inequality

$$
\operatorname{vol} N \geqq C_{0} \cdot 4 \pi=\operatorname{vol} \Delta_{0}=3.66386 \ldots,
$$

where the equality holds iff $N$ admits a polyhedral decomposition by a regular truncated tetrahedra of ridge length $=0$.

When $N$ has no $\partial$-cusps, $\ell_{N}$ is a non-zero constant. Then by an argument in [15], we can pack two disks of radius $R_{N}$ in $\partial N$ where

$$
\cosh ^{2} R_{N}=\frac{2 \cosh \ell_{N}-1}{2\left(\cosh \ell_{N}-1\right)} .
$$

If $\ell_{N}$ is short, then $R_{N}$ becomes long and more area on the boundary is needed. Hence with the help of Böröczky's bounds for packing density in [1], we can estimate $\ell_{N}$ by area $\partial N$,

$$
\begin{aligned}
2 \cdot 2 \pi\left(\cosh R_{N}-1\right) & \leqq \text { area } \partial N \cdot \text { Böröczky's bound } \\
& =\operatorname{area} \partial N \cdot \frac{3 \theta_{N}\left(\cosh R_{N}-1\right)}{\pi-3 \theta_{N}},
\end{aligned}
$$

which is simplified to

$$
\frac{\pi}{3(1-\chi(\partial N) / 2)} \leqq \theta
$$

Then, fixing the topology of the boundary, we can refine the theorem by 
Corollary. If $N$ has no $\partial$-cusp, then

$$
\operatorname{vol} N \geqq(1-\chi(\partial N) / 2) \cdot \operatorname{vol} \Delta_{\pi / 3(1-\chi(\partial N) / 2)} \text {. }
$$

The equality holds iff $N$ admits a decomposition by $\Delta_{\pi / 3(1-\chi(\partial N) / 2)}$ 's.

With a little argument, we also rediscover the compact smallest $N^{\prime} s$.

CoRollary ([15]). If $N$ is compact, then

$$
\operatorname{vol} N \geqq 2 \operatorname{vol} \Delta_{\pi / 6}=6.45199 \ldots \text {, }
$$

where the equality holds iff $N$ admats a decomposition by two $\Delta_{\pi / 6}$ 's.

Thus the smallest values became to be fairly accessible, though their number theoretic properties are still almost unknown. Another extremal values, such as the first limit cardinal, seems to be completely unknown either.

\section{Reference}

[1] K. Böröczky, Packing of spheres in spaces of constant curvature, Acta Math. Acad, Sci. Hungar., 32 (1978), 243-261.

[2] S. Ballett and G. Montica, Group theory of hyperbolic circle packings, Nonlinearity, 5 (1992), 1085-1109.

[ 3 ] R. Benedetti and C. Petronio, Lectures on hyperbolic geometry, Universitext, Springer-Verlag, 1992.

[4] D. Bessis and S. Demko, Generalized Apollonian packings, Commun. Math. Phys., 134 (1990), 293-319.

[ 5 ] R. Canary, D. Epstein and P. Green, Notes on note of Thurston, LMS Lecture Notes Series, 111 (1987), 3-92.

[6] D. Epstein and R. Penner, Euclidean decompositions of noncompact hyperbolic manifolds, J. Differential Geom., 27 (1988), 67-80.

[ 7 ] M. Fujii, Hyperbolic 3-manifolds with totally geodesic boundary which are decomposed into hyperbolic truncated tetrahedra, Tokyo J. Math., 13 (1990), 353-373.

[8] M. Fujii, On totally geodesic boundaries of hyperbolic 3-manifolds, Kodai Math. J., 15 (1992), 244-257.

[ 9 ] M. Fujii, in preparation.

[10] M. Ishida and S. Kojima, Apollonian packings and hyperbolic geometry, Geom. Dedicata, 43 (1992), 265-283.

[11] R. Kellerhals, On the volume of hyperbolic polyhedra, Math. Ann., 285 (1989), 541-569.

[12] S. Kojima, Polyhedral decomposition of hyperbolic manifolds with boundary, Proc. Workshops Pure. Math., 10 (1990), 37-57.

[13] S. Kojima, Polyhedral decomposition of hyperbolic 3-manifolds with totally geodesic boundary, Adv. Studies Pure Math., 20 (1992), 93-112.

[14] S. Kojima, Lectures at Univ. of Tokyo, 1991.

[15] S. Kojima and Y. Miyamoto, The smallest hyperbolic 3-manifolds with totally geodesic boundary, J. Differential Geom., 34 (1991), 175-192.

[16] Y. Miyamoto, On the volume and surface area of hyperbolic polyhedra, Geom. Dedicata, 40 (1991), 223-236.

[17] Y. Miyamoto, Volumes of hyperbolic manifolds with geodesic boundary, to appear in Topology.

[18] G. Mostow, Quasi-conformal mappings in $n$-space and the rigidity of hyperbolic space forms, Publ. IHES, 34 (1973), 53-104. 
[19] W. Neumann and A. Reid, Rigidity of cusps in deformations of hyperbolic 3-orbifolds, Math. Ann., 295 (1993), 223-237.

[20] G. Prasad, Strong rigidity of Q-rank 1 lattices, Inventiones math., 21 (1973), 255-286.

[21] W. Thurston, The geometry and topology of 3-manifolds, Lecture Notes, Princeton Univ. $(1977 / 78)$.

Department of Mathematical and

COMPUTING SCIENCES

TOKYO INSTITUTE OF TECHNOLOGY

OHOKAYAMA, MEguro

TOKYO 152 JAPAN

e-mail: sadayosi@is.titech.ac.jp 